\title{
Overexpression of chalcone synthase gene improves flavonoid accumulation and drought tolerance in tobacco
}

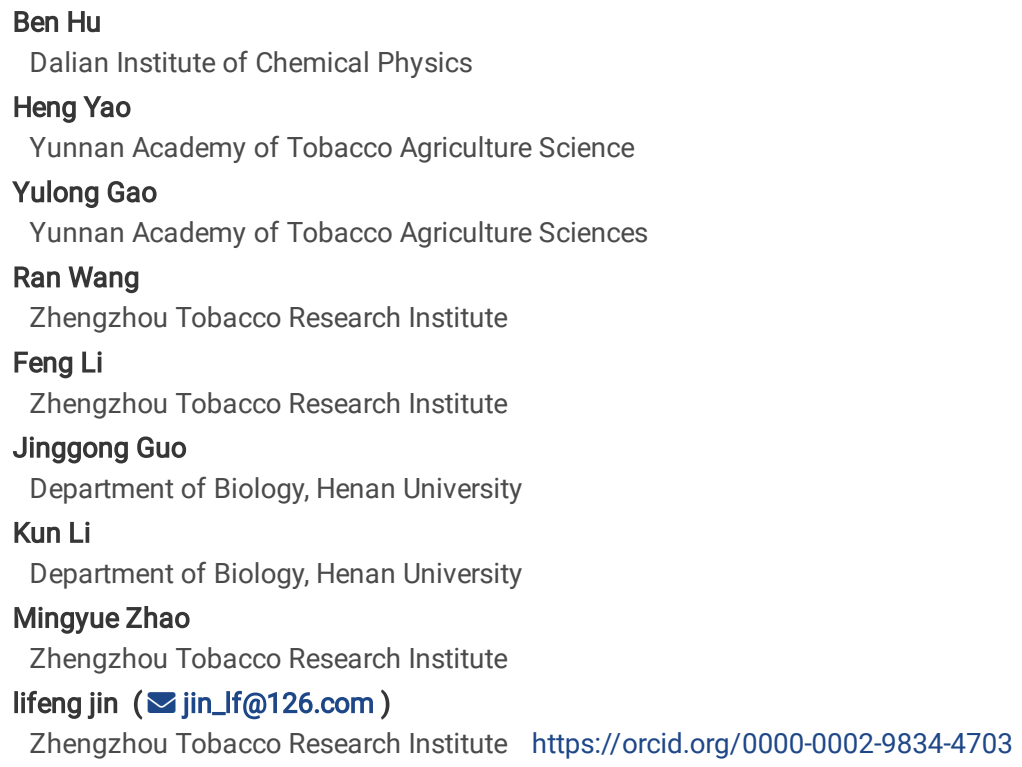




\section{Abstract}

Flavonoids are important secondary metabolites in plants that play important roles in maintaining the cellular redox balance of cells. Chalcone synthase (CHS) is the key enzyme in the flavonoid biosynthesis pathway and has been found to monitor changes due to drought stress tolerance. In this study, a $\mathrm{CHS}$ gene in tobacco (Nicotiana tabacum ) was overexpressed. Results revealed that transgenic tobacco plants were more tolerant than control plants to drought stress. Transcription levels of the key genes involved in the flavonoid pathway and the contents of seven flavonoids significantly increased in transgenic tobacco plants ( $p$ < 0.01). Overexpression of the CHS gene led to lower concentrations of the oxidative stress product, malondialdehyde (MDA). Additionally, $11 \mathrm{CHS}$ family genes were mined from the tobacco genome. Based on the phylogenetic tree, these genes split into two groups with eight genes clustered together with the bona fide Arabidopsis CHS gene, suggesting that those tobacco genes are CHS genes. Further phylogenetic analyses indicated that the tobacco CHS genes grouped further into three independent clades with the cloned tobacco CHS gene located within Clade iii. The tobacco CHS family genes exhibited a highly conserved CDS length, $\mathrm{pl}$, and molecular weight of the encoded peptides. All CHS peptides contained two conserved domains, and the genes harbored two or three exons. Based on the results of this study, the NtCHS gene is considered a possible candidate gene for genetically engineering enhanced drought tolerance and improved responses to oxidative stress in plants.

\section{Introduction}

Flavonoids are a group of plant secondary metabolites consisting of two aromatic rings linked by three carbons. Flavonoids play diverse roles in plants, including the control of floral pigmentation, protecting plants from ultraviolet (UV) damage, and regulating the polar transport of auxin. Moreover, flavonoids play important roles in the cellular response to biotic and abiotic stressors. ${ }^{1}$ To date, the structure and function of many enzymes in the flavonoid pathway have been well characterized in plants, including chalcone synthase (CHS), chalcone isomerase (CHI), flavanone 3-hydroxylase (F3H), dihydroflavonol 4reductase (DFR), flavonol synthase (FLS), and anthocyanidin synthase (ANS). ${ }^{2,3}$

Recent studies have investigated the effect of drought stress on flavonoid metabolism in plants, and it has been demonstrated that flavonoids accumulate rapidly in some plant species suffering from drought stress. ${ }^{4}$ For example, winter wheat leaves exhibit increased expression levels of key flavonoid genes and total flavonoid content in response to drought stress. ${ }^{5}$ In the leaves of Ziziphus jujuba var. spinose, the total flavonoid content increased significantly under moderate drought treatment. ${ }^{6}$ In the roots of Scutellaria baicalensis georigi, the expression levels of several flavonoid genes were upregulated under drought conditions. ${ }^{7}$ In short, evidence thus far indicates the participation of flavonoids and their key biosynthesis genes in plant resistance to drought stress. However, little is known about the exact roles of these key flavonoids genes in plant responses to drought stress.

The CHS gene encodes the first enzyme in the flavonoid pathway, and the CHS enzyme catalyzes the synthesis of narengenin chalcone by using one molecule of p-coumaryl-CoA and three molecules of malonyl-CoA, which are retrieved from the phenylpropanoid pathway. ${ }^{3} \mathrm{CHS}$ is believed to have evolved from primary metabolic enzymes involved in fatty acid biosynthesis. ${ }^{2}$ Many $\mathrm{CHS}$ genes have been cloned from higher plants, and their structures have been wellstudied. ${ }^{8-10} \mathrm{CHS}$ proteins exhibit diverse functions among different plants, including anti-oxidant activity, participating in floral pigment formation, ${ }^{11-13}$ as well as pollen fertility. ${ }^{14}$ However, little research has focused on the role of plant $\mathrm{CHS}$ gene expression levels in drought tolerance or in regulating the expression patterns of downstream genes and metabolite concentrations.

This study aimed to investigate the role of the tobacco (Nicotiana tabacum) CHS (NtCHS) gene in response to drought conditions. A vector overexpressing the $\mathrm{NtCHS}$ coding sequence was constructed and transferred into tobacco. Transgenic plants exhibited increased flavonoid contents, and the expression levels of relevant genes were upregulated. Collectively, these responses enhanced drought tolerance in transgenic plants. Additionally, $\mathrm{CHS}$ family genes were mined from the tobacco genome, which can serve as a reference for future studies on tobacco CHS genes.

\section{Materials And Methods}

\subsection{Chemicals and reagents}

Seven flavonoid reference standards (i.e., rutin, quercetin, kaempferol-3-rutinoside, kaempferol-glucopyranoside, naringin, naringenin, and isoliquiritigenin) and the internal standard vitexin (purity $\geq 98 \%$ ) were bought from Yudingxinjie Corporation (Zhengzhou, China). Acetonitrile, methanol, ethanol, and formic acid of high-performance liquid chromatography (HPLC) grade were purchased from J.T. Baker (WI, USA). Ultra-pure water was prepared with a Milli-Q purification system (Millipore, MA, USA). Thiobarbituric acid and titanium sulfate ( $\geq 98 \%$ ) were obtained from Yudingxinjie Corporation (Zhengzhou, China).

\subsection{Vector construction, plant transformation, and confirmation}

NtCHS cDNA (accession No.: AF311783.1) was amplified using two primers, NtCHSF 5'-AGCCATTTGAAAACCCTAG-3' and NtCHSR 5'-

CAAATTTCATTATTTGCAA G-3'. Then, NtCHS cDNA was cloned into the pH7WG2D plasmid between the attR1 and attR2 sites, which was confirmed by sequencing (Fig. 1a). Afterward, a recombinant vector pH7WG2D-NtCHS was prepared and transformed into the Agrobacterium strain, EHA105. N. tabacum K326 was transformed using a leaf disk method following standard protocol (Fig. 1a). ${ }^{15}$

Seeds were collected from self-pollinated primary transformants. Transgenic tobacco seeds $\left(T_{1}\right.$ generation) were germinated with $50 \mathrm{mg} \mathrm{mL}^{-1}$ kanamycin. Seeds of transgenic and control tobacco lines were grown in pots containing a mixture of manure, sand, and soil (1:1:2) in a greenhouse. The transformed tobacco plants were screened to identify Npt //via polymerase chain reaction (PCR) using genomic DNA as the template and two primers, $n p t$ IIF $5^{\prime}$ TGTCACTGAAGCGGGAAG-3' and npt II-R 5'-CTTCCATCCGAGTAC GTG-3'. The PCR conditions were as follows: 1 min at $94^{\circ} \mathrm{C}$; 35 cycles for $30 \mathrm{~s}$ at $94^{\circ} \mathrm{C}, 30 \mathrm{~s}$ at $50^{\circ} \mathrm{C}$, and $1 \mathrm{~min}$ at $72^{\circ} \mathrm{C}$. PCR products were separated by agarose gel electrophoresis and visualized with ethidium bromide staining and UV illumination. 
Genomic DNA was isolated from the pH7WG2D-NtCHS transformants and control plants using a DNeasy plant mini kit (Qiagen, Duesseldorf, Germany) following the manufacturer's instructions.

To select positive transgenic tobaccos, NtCHS gene expression was determined by fluorescent real-time quantitative PCR (RT-qPCR). For each plant, 100 mg sample was ground in liquid nitrogen. Then, total RNA was extracted from the pH7WG2D-NtCHS transformants and control plants separately using an RNeasy plant mini kit (Qiagen, Duesseldorf, Germany) following the manufacturer's instructions. After treatment with DNase I (GeneAnswer, Zhengzhou, China), firststrand CDNA was synthesized using avian myeloblastosis virus reverse transcriptase (Sangon, Shaanghai, China) following the manufacturer's instructions. RT-qPCR was performed on a CFX96 instrument (BIO-RAD, CA, USA). Each reaction mixture (total volume $=20 \mu \mathrm{L})$ contained $10 \mu \mathrm{L}$ SYBR Premix Ex Taq $(2 \times$ concentration) (TaKaRa, Dalian, China), $1 \mu \mathrm{L}$ gene-specific primers CHSF/CHS-R (10 mM), and diluted cDNA (100 ng). Leaf samples of both CHSoverexpressing tobacco and control plants were tested in triplicate. Amplification conditions were as follows: $30 \mathrm{~s}$ at $95^{\circ} \mathrm{C} ; 40 \mathrm{cycles}$ for $5 \mathrm{~s}$ at $95^{\circ} \mathrm{C}, 40 \mathrm{~s}$ at $60^{\circ} \mathrm{C}$; one cycle for $15 \mathrm{~s}$ at $95^{\circ} \mathrm{C}, 1 \mathrm{~min}$ at $60^{\circ} \mathrm{C}, 15 \mathrm{~s}$ at $95^{\circ} \mathrm{C}, 15 \mathrm{~s}$ at $60^{\circ} \mathrm{C}$. The internal standard gene was the $26 \mathrm{~S}$ rRNA-based gene primer. ${ }^{16} \mathrm{No}$ morphological differences were observed between the transgenic and control plants.

\subsection{Plant growth conditions and drought treatment}

Seeds were grown on soil or one-half-strength Murashige and Skoog (MS) agar medium in a growth chamber maintained at $22-24^{\circ} \mathrm{C}$ and $60 \%$ relative humidity under a $16 / 8 \mathrm{~h}$ light/dark photoperiod. After germination with $50 \mathrm{mg} \mathrm{mL}^{-1}$ kanamycin, seedlings of transgenic tobacco seeds were transferred to culture pots in the greenhouse where the drought treatment was applied for $14 \mathrm{~d}$. Fresh weight (FW) of leaves was measured after removal from plants. Turgid weight (TW) was determined after rinsing the leaves in water at $4^{\circ} \mathrm{C}$ for $12 \mathrm{~h}$. Then, the dry weight (DW) of leaves was determined after drying at $80^{\circ} \mathrm{C}$ for $48 \mathrm{~h}$. Relative water content (RWC) was calculated as RWC $=[(\mathrm{FW}-\mathrm{DW}) /(\mathrm{TW}-\mathrm{DW})] \times 100 \% .{ }^{17} \mathrm{H}_{2} \mathrm{O}_{2}$ content in transgenic and control tobacco plants was measured after the drought treatment concluded. ${ }^{18}$ Content of malondialdehyde (MDA) was detected using the thiobarbituric acid (TBA) method. ${ }^{19}$

\subsection{RNA isolation and RT-qPCR}

Total RNA was extracted from the leaves of CHS-overexpressing tobacco and control plants using an RNeasy plant mini kit (Qiagen, Duesseldorf, Germany). Then, first-strand cDNA was synthesized. The aforementioned RT-qPCR conditions and internal standard gene were used here. The forward and reverse primers used for RT-qPCR were as follows: CHS, 5'-AGAAAAGCCTTGTGGAAGCA-3', 5'-ACTTGGTCCAAAATTGCAGG-3'; CHI, 5' -GAAATCCTCCGATCCAGTGA-3', 5'-CAACGTTGACAACATCAGGC-3'; F3H, 5'-ACAGGGTGAAGTGGTCCAAG-3', 5' -CCTTGGTTAAGGCCTCCTTC-3'; F3'H, 5'-TCCAAGAATACTGGCCCAAG-3', 5'CTCACAACTCTCGGATGCAA-3'; FLS, 5'-GAACTTGAAGGGAAAAGGGG-3', 5'-TCCCTGTAGGAGGGAGGATT-3'; DFR, 5'-TCCCATCATGCGATCATCTA-3', 5' ATGGCTTCTTTGTCACGTCC-3'; and, 26S rRNA, 5'-CACGGACCAAGGAGTCTGACAT-3', 5'-TCCCAC CAATCAGCTTCCTTAC-3'.

\subsection{Liquid chromatography and tandem mass spectrometry}

Flavonoids were extracted from tobacco plants following standard protocol with minor modifications. ${ }^{20}$ Freeze-dried tobacco powder (50 mg) and $2 \mathrm{~mL}$ water/methanol extract

Fig. 1 Schematic map of the tDNA region of pH7WG2D-NtCHS and confirmation of successful transformation. (a) schematic representation of ph7wg2d harboring the $n t c h s$ cdna. (b) pcr using genomic DNA as the template confirms the introduction of $n p t / /$ into the transgenic tobacco line. Npt // is absent in the control tobacco lines, C1, C2, and C3.

solution (4:1) were mixed and sonicated in an ultrasonic bath at $30^{\circ} \mathrm{C}$ for $20 \mathrm{~min}$. Then, the extracts were centrifuged at $4000 \mathrm{rpm}$ for $20 \mathrm{~min}$, and the supernatants were collected. Seven flavonoids (i.e., rutin, quercetin, kaempferol-3-rutinoside, kaempferol-glucopyranoside, naringin, naringenin, and isoliquiritigenin) were identified and quantified by using a QQQ 6490 high-performance liquid chromatography mass spectrometry (HPLC-MS) system (Agilent, $\mathrm{CA}, \mathrm{USA})$ and electrospray ionization operated in positive ion mode $\left(\mathrm{ESI}^{+}\right)$. The flavonoids were separated on a zorbax sb-c18 column (100 $\times 2.1 \mathrm{~mm}{ }^{2}$; particle size, $1.8 \mathrm{~m}$ ) (Agilent, CA, USA) at $30^{\circ} \mathrm{C}$. The mobile phase consisted of water/formic acid (A) (99.9:0.1) and acetonitrile/formic acid (B) (99.9:0.1) at a flow rate of $0.3 \mathrm{~mL} \mathrm{~min}-1$. Optimal separation was achieved with a gradient elution of $95 \% A$ and $5 \%$ B in the beginning maintained for 2 min, changed to $75 \% A$ for 10 $\min$, and $5 \%$ A for $12 \mathrm{~min}$. The injection volume was $3 \mu \mathrm{L}$.

The optimized MS parameters were as follows: drying gas at $350^{\circ} \mathrm{C}$; capillary voltage $4.0 \mathrm{kV}$; drying gas flow rate $11 \mathrm{~L}$ min $^{-1}$; nebulizer pressure 45 psi. The reaction-monitoring mode with $\mathrm{ESI}^{+}$was $\mathrm{m} / \mathrm{z} 449.1 \rightarrow 287.0$ for kaempferol-glucopyranoside, $\mathrm{m} / \mathrm{z} 581.2 \rightarrow 273.1$ for naringin, $\mathrm{m} / \mathrm{z} 273.1 \rightarrow 153.1 \mathrm{for}$ naringenin, $\mathrm{m} / \mathrm{z} 257.1 \rightarrow 137.1 \mathrm{for}$ isoliquiritigenin, and $\mathrm{m} / \mathrm{z} 433.1 \rightarrow 313.1 \mathrm{for}$ the internal standard, vitexin. Flavonoids were quantified by referring to the standard curves of the seven flavonoids and vitexin dissolved in methanol/water (4:1). Instrument control, data acquisition, and evaluations were completed with MassHunter Agilent 2003-2007 Data Acquisition for Triple Quad B.01.04 (B84).

\subsection{Mining of CHS family genes and bioinformatics analyses}

CDS and peptide sequence datasets were downloaded for Arabidopsis, tomato, coffee, tobacco (N. tabacum), and N. attenuata. Datasets were preprocessed to clean sequences and accession lines. ${ }^{21-25}$ The cloned Arabidopsis and tobacco CHS genes were used for retrieving peptides with the same conserved domains using HMMER against a library of Pfam-A families with default parameters. ${ }^{26} \mathrm{CDS}$ sequences were retrieved from CDS datasets based on the locus IDs of peptide sequences. Peptide sequences were aligned using Probcons v1.12. ${ }^{27}$ If required, the file formats of aligned sequences were converted to phy. Maximum-likelihood phylogenetic trees were reconstructed using PhyML using a bootstrap method with 1,000 replicates. ${ }^{28}$ Unless otherwise indicated, default parameters of phylogeny inference were used. Phylogenetic trees were visualized with FigTree v1.3.1. 


\section{Results}

\subsection{Generation of $\mathrm{NtCHS}$ overexpressing tobacco plants}

An overexpression construct, pH7WG2D-NtCHS, with NtCHS cDNA (AF311783.1) under the control of the CaMV35S promoter was transformed into N. tabacum K326 (Fig. 1a). Three independent putative positive lines were selected on the MS medium with kanamycin. Positive lines were further confirmed with genomic PCR using the primer pair, npt IFF and npt IIR. The PCR results revealed that the $n p t / /$ gene was expressed in the three selected transgenic lines (i.e., F1, F2, and F3), but not in the control plants (i.e., C1, C2, and C3) (Fig. 1b). Since the empty vector, pH7WG2D, contained a suicide gene, ccdB, between the attR1 and attR2 sites, the plasmid pH7WG2D-NtCHS was successfully transferred into tobacco plants. Transgenic lines were further selected by kanamycin resistance for subsequent analyses.

\subsection{Expression patterns of flavonoid genes in transgenic tobacco}

In order to investigate the expression patterns of the flavonoid genes, leaves at the vigorous growing stage were harvested from transgenic and control plants. The three transgenic tobacco lines exhibited significantly higher NtCHS transcription levels compared to the control plants $(p<0.01)$. Therefore, they were selected for subsequent experimentation (Fig. 2a). Moreover, the expression levels of the $C H I, F 3 H, F 3^{\prime} H, F L S$, and $D F R$ genes in transgenic tobacco plants were significantly higher compared to the control tobacco plants $(p<0.01)$.

\subsection{Flavonoid accumulation in tobacco leaves}

In order to investigate the effect of the NtCHS gene on flavonoid biosynthesis in tobacco, flavonoid content in transgenic and control leaves was detected. The content of all flavonoids was much higher in transgenic plants compared to control plants (Table 1, Fig. 3).

Table 1 Quantification of seven flavonoids in transgenic and control tobacco lines by HPLC-MS ESI ${ }^{+}$

\begin{tabular}{|c|c|c|c|c|c|c|}
\hline \multirow[t]{2}{*}{ Flavonoids } & \multicolumn{6}{|c|}{ Dry weight $\left(\mathrm{mg} \mathrm{g}^{-1} ;\right.$ mean \pm S.E. $)$} \\
\hline & $\mathrm{C} 1$ & $\mathrm{C} 2$ & C3 & F1 & F2 & F3 \\
\hline Rutin & $\begin{array}{c}1.2095 \pm \\
0.3667\end{array}$ & $\begin{array}{c}0.8526 \pm \\
0.1499\end{array}$ & $\begin{array}{c}0.7171 \pm \\
0.3721\end{array}$ & $\begin{array}{l}9.8294 \pm \\
1.2524\end{array}$ & $\begin{array}{c}24.1283 \pm \\
3.2131\end{array}$ & $\begin{array}{c}42.5386 \pm \\
14.4964\end{array}$ \\
\hline Quercetin & $\begin{array}{c}0.4346 \pm \\
0.1447\end{array}$ & $\begin{array}{c}0.7190 \pm \\
0.0708\end{array}$ & $\begin{array}{c}0.5373 \pm \\
0.2265\end{array}$ & $\begin{array}{l}7.5818 \pm \\
4.2555\end{array}$ & $\begin{array}{l}10.2958 \pm \\
2.6337\end{array}$ & $12.1381 \pm 5.4301$ \\
\hline Naringenin & $\begin{array}{c}0.0333 \pm \\
0.0138\end{array}$ & $\begin{array}{c}0.0371 \pm \\
0.0063\end{array}$ & $\begin{array}{c}0.0345 \pm \\
0.0041\end{array}$ & $\begin{array}{c}0.7983 \pm \\
0.3606\end{array}$ & $0.4717 \pm 0.0425$ & $0.5750 \pm 0.0913$ \\
\hline Kaempferol-3-rutinoside & $\begin{array}{c}0.0240 \pm \\
0.0034\end{array}$ & $\begin{array}{c}0.2122 \pm \\
0.0268\end{array}$ & $\begin{array}{c}0.0493 \pm \\
0.0197\end{array}$ & $\begin{array}{c}7.4321 \pm \\
0.6083\end{array}$ & $\begin{array}{c}12.1679 \pm \\
2.0946\end{array}$ & $16.0063 \pm 3.4738$ \\
\hline $\begin{array}{l}\text { Kaempferol- } \\
\text { glucopyranoside }\end{array}$ & ND & $\begin{array}{c}0.1032 \pm \\
0.0131\end{array}$ & ND & $\begin{array}{c}1.4398 \pm \\
0.3965\end{array}$ & $0.8898 \pm 0.1120$ & $1.0927 \pm 0.3114$ \\
\hline Naringin & ND & ND & ND & $\begin{array}{c}1.3620 \pm \\
0.4234\end{array}$ & $1.4423 \pm 0.1459$ & $0.9575 \pm 0.1395$ \\
\hline Isoliquiritigenin & $\begin{array}{c}0.0108 \pm \\
0.0030\end{array}$ & $\begin{array}{c}0.0132 \pm \\
0.0024\end{array}$ & $\begin{array}{c}0.0105 \pm \\
0.0016 \\
\end{array}$ & $\begin{array}{c}0.0236 \pm \\
0.0067 \\
\end{array}$ & $0.0170 \pm 0.0023$ & $0.0268 \pm 0.0039$ \\
\hline
\end{tabular}

ND, not detected $(n=3)$.

\subsection{Drought tolerance of tobacco plants}

In order to evaluate whether NtCHS overexpression affects tobacco drought tolerance, a water-deficit treatment was performed. The growth of transgenic plants was notable better

Fig. 2 Abundance of the transcripts of flavonoid transcriptional genes in transgenic and control tobacco lines measured by RT-qPCR: (a) $\mathrm{CHS}$, (b) $\mathrm{CHI}$, (c) F3H, (d) $F 3^{\prime} H$, (e) $F L S$, and (f) $D F R$. ${ }^{*} p<0.05 ; * \star p<0.01$.

compared to control plants after $14 \mathrm{~d}$ (Fig. 4a). To further characterize the performance of transgenic tobacco plants under drought stress, changes in the concentrations of RWC, MDA, and $\mathrm{H}_{2} \mathrm{O}_{2}$ were monitored before and after drought treatment. After $14 \mathrm{~d}$ of water shortage, the RWCs of the three transgenic lines were significantly higher compared to the control plants $(p<0.01)$ (Fig. 4b). Leaf MDA concentrations also increased in transgenic and control tobacco plants following drought stress (Fig. 4c). The concentrations of ROS were not significantly different under drought treatment conditions. However, control tobacco plants had higher concentrations of $\mathrm{H}_{2} \mathrm{O}_{2}$ compared to transgenic plants under drought treatment conditions (Fig. 4d).

Fig. 3 Quantification of seven flavonoids in transgenic and control tobacco lines by HPLC-MS ESI ${ }^{+}$.

\subsection{Mining of $\mathrm{CHS}$ family genes in the tobacco genome}

Because cDNA we cloned and identified had a role in flavonoid accumulation and drought tolerance, how this gene evolved was investigated and whether there were other $\mathrm{CHS}$ genes that possess potential relevant functions was explored. Using HMMER with the known tobacco $\mathrm{CHS}$ gene (accession No.: AF311783.1) as a query, 11 peptides were mined from the tobacco genome, all of which contained two conserved domains (i.e., Pfam ID: PF02797.14 and PF00195.18), which are signatures of CHS peptides. One of the mined peptides (accession No. XP_016480648.1) exhibited 100\% identity with the gene cloned in this study (AF311783.1), suggesting that the two genes are virtually the same. Additionally, peptides were mined from the genomes of $N$. attenuate $(n=7)$, tomato $(n=7)$, coffee $(n=10)$, and Arabidopsis $(n=4)$. In total, 35 peptides were mined from four Solanaceae genomes.

\subsection{Phylogenetic relationships of $\mathrm{CHS}$ peptides}


In order to determine the phylogenetic relationships among the mined genes, 39 peptide sequences were aligned and used as input to infer the phylogenetic tree. The resulting tree revealed that the peptides were split into two distinct groups (Fig. 5a). Identity values among the genes within their respective groups were generally considerably higher than those between the two groups (Table 2). Seven of the tobacco peptides clustered together with a bona fide Arabidopsis CHS gene (AT5G13930.1) (Fig. 5a, I), while four peptides grouped with three other Arabidopsis peptides, one of which was previously reported to be hydroxyalkyl a-pyrone synthase. ${ }^{29,30}$ Therefore, genes in Class I of the mined sequences putatively encode CHSs (Fig. 5a).

To better resolve CHS phylogeny, the sequences in Class I were retrieved after globally aligned results were used for phylogenetic inference, which revealed that the Solanaceae CHS genes further split into three independent clades with high branch bootstrap support (i.e., Clades i, ii, and iii) (Fig. 5b). In each clade, no less than two tobacco genes were present, while only one tomato copy was present, which was in agreement with the haploid nature of the tomato genome compared to the tetraploid nature of the tobacco genome. The cloned gene clustered in Clade iii (XP_016480648.1), which was phylogenetically close to another tobacco gene (XP_016494384.1) and one tomato gene (Solyc09g091510.2.1) (Fig. 5b). The two tobacco CHS peptide sequences in Clade iii exhibited a high identity value (98.7\%). High identity values were also observed in tobacco CHSs within the other two clades (Table 2).

Table 2 Identity values (\%) between peptide sequences of tobacco CHSs

\begin{tabular}{|c|c|c|c|c|c|c|c|}
\hline & XP_016457437.1 & XP_016482301.1 & XP_016446648.1 & XP_016494384.1 & XP_016480648.1 & XP_016494186.1 & $\overline{\mathrm{XP} \_016 !}$ \\
\hline XP_016514139.1 & 97.8 & 67.4 & 67.4 & 40.6 & 41.2 & 39.2 & $\overline{3 !}$ \\
\hline XP_016457437.1 & & 67.7 & 67.9 & 40.6 & 41.2 & 39.2 & $3 !$ \\
\hline XP_016482301.1 & & & 97.0 & 41.2 & 41.7 & 38.9 & $3\}$ \\
\hline XP_016446648.1 & & & & 40.4 & 40.9 & 38.1 & $3 i$ \\
\hline XP_016494384.1 & & & & & 98.7 & 76.2 & $7 !$ \\
\hline XP_016480648.1 & & & & & & 76.2 & $7 !$ \\
\hline XP_016494186.1 & & & & & & & $9<$ \\
\hline XP_016515539.1 & & & & & & & \\
\hline XP_016508885.1 & & & & & & & \\
\hline
\end{tabular}

\subsection{Characteristics of the tobacco CHS family genes and encoded peptides}

The 11 tobacco genes were distributed in different scaffolds of the tobacco genome, which may be due to low assembly quality. However, the CDS length (1167-1293 bp), pl values (5.57-8.02), and molecular weights (42.5-47.4 kDa) were highly similar among the genes and their encoded peptides, which represent conserved sequence characteristics among the 11 tobacco genes (Table 3). All peptide sequences contained two conserved domains, with PF00195.18 located at the N-terminus and PF02797.14 at the C-terminus (Fig. 6a). All CDS sequences of the CHS family genes contained one or two introns with highly variable lengths, and the first exon of the genes tended to be considerably shorter than the second and third exons (Fig. 6b).

Table 3 Characteristics of tobacco CHS family genes and encoded proteins

\begin{tabular}{lccccccc}
\hline ID & Scaffold & Start-end & Strand & CDS_length (bp) & pI & MW (kDa) & Exons \\
\hline XP_016514139.1 & NW_015858924.1 & $39284-40989$ & - & 1185 & 5.63 & 43.8 & 2 \\
\hline XP_016457437.1 & NW_015907297.1 & $47081-48556$ & - & 1185 & 6.55 & 43.7 & 2 \\
\hline XP_016482301.1 & NW_015802956.1 & $73048-75966$ & + & 1170 & 5.57 & 43.2 & 3 \\
\hline XP_016446648.1 & NW_015891785.1 & $63744-67497$ & - & 1170 & 6.28 & 43.2 & 3 \\
\hline XP_016494384.1 & NW_015806518.1 & $130722-133092$ & - & 1167 & 6.71 & 42.6 & 2 \\
\hline XP_016480648.1 & NW_015934644.1 & $52313-54708$ & + & 1167 & 6.71 & 42.6 & 2 \\
\hline XP_016494186.1 & NW_015806028.1 & $38323-40241$ & + & 1170 & 6.52 & 42.8 & 3 \\
\hline XP_016515539.1 & NW_015863094.1 & $82043-85472$ & - & 1170 & 6.32 & 42.8 & 2 \\
\hline XP_016480039.1 & NW_015933844.1 & $25918-28090$ & - & 1293 & 8.02 & 47.4 & 2 \\
\hline XP_016508885.1 & NW_015843977.1 & $4364-6561$ & - & 1167 & 6.71 & 42.5 & 2 \\
\hline XP_016489904.1 & NW_015948736.1 & $40753-43241$ & + & 1167 & 6.51 & 42.5 & 2 \\
\hline
\end{tabular}

Fig. 4 (a) Qualitative observations of the drought experiment. (b) RWC in transgenic and control tobacco lines. (c) Concentrations of MDA in transgenic and control tobacco lines. (d) Concentrations of $\mathrm{H}_{2} \mathrm{O}_{2}$ in transgenic and control tobacco lines. $\mathrm{C} 1-\mathrm{C} 3$ : control tobacco lines; $\mathrm{F} 1-\mathrm{F} 3$ : $\mathrm{T}_{1}$ generation transgenic tobacco lines. ${ }^{*} p<0.05 ; * \star p<0.01$.

Fig. 5 Phylogeny of CHS family peptides. CHS family peptide sequences were retrieved from tobacco, tomato, Arabidopsis, coffee, and N. attenuata. PhyML was used for the reconstruction of phylogenetic trees. (a) Unrooted phylogenetic tree of CHS family genes showing two independent groups (circled and labeled with I and II, respectively). (b) Phylogenetic tree of CHS genes. Red labels indicate tobacco genes, green labels indicate Arabidopsis genes. Clades i, ii, and iii are shaded in light green, purple, and green, respectively.

\section{Discussion}

The flavonoid biosynthesis pathway is an important secondary metabolite pathway, and CHS is the first committed enzyme that catalyzes the synthesis of the flavonoid branch. Previous work suggested a role for CHS in the production of flavonoids and improving plant tolerance to drought stress. ${ }^{13,31,32}$ In this study, overexpression of NtCHS in tobacco plants enhanced plant tolerance to drought stress and increased RWC. Additionally, transcripts of the flavonoid biosynthesis genes and flavonoid contents were considerably upregulated in transgenic plants. Four of the seven measured flavonoids (i.e., rutin, quercetin, kaempferol-3-rutinoside, and kaempferol-glucopyranoside) were higher in content, ${ }^{33,34}$ while naringin, naringenin, and isoliquiritigenin occupied key positions in the metabolic pathway. Overall, these results suggest that flavonoids play a role in plant drought tolerance. ${ }^{5,7}$ 
Exposure to drought conditions can result in the increased production of ROS, oxidative stress, and cell membrane damage in plants. ${ }^{35}$ Flavonoids affect plant physiology in response to external stressors and play an important role in the maintenance of cellular redox balance. ${ }^{36}$ It has been demonstrated that flavonoids prevent the formation of ROS and improve the scavenging of ROS in drought-stressed plants. ${ }^{37-39}$ Before exposure to drought conditions, the concentrations of MDA and $\mathrm{H}_{2} \mathrm{O}_{2}$ in transgenic plants were similar to control plants. However, transgenic tobacco plants possessed lower concentrations of ROS and MDA than control plants when exposed to drought stress conditions. MDA is a lipid peroxidation product that is produced due to drought stress in plants. ${ }^{40}$ Therefore, the results of this study suggest that the measured flavonoids (i.e., rutin, quercetin, kaempferol-3-rutinoside, kaempferol-glucopyranoside, naringin, naringenin, and isoliquiritigenin) play a role in maintaining the redox balance of transgenic tobacco in response to drought stress, as indicated by the lower concentrations of MDA.

By using the $14 \mathrm{~d}$ drought tolerance treatment, it was demonstrated that the overexpression of the NtCHSgene in tobacco resulted in improved plant drought tolerance. Additionally, the transcripts of key flavonoid pathway genes and contents of seven flavonoids significantly increased in transgenic plants.

Furthermore, concentrations of ROS were lower in transgenic tobacco plants than control plants, suggesting a role for flavonoids in maintaining cellular redox balance. The results of this study suggest that $\mathrm{NtCHS}$ is a potential candidate gene that could be targeted in the genetic engineering of tobacco plants in order to enhance drought stress tolerance.

In addition to the cloned tobacco $\mathrm{CHS}$ gene, $\mathrm{CHS}$ genes were systematically mined from the tobacco genome, leading to the identification of $11 \mathrm{CHS}$ family genes. Phylogenetic analyses indicated that there are $8 \mathrm{CHS}$ genes in the tobacco genome, which were derived from two rounds of duplications in Solanaceae. The genes exhibited a conservation of sequence characteristics, indicating that these genes are conserved in biological and enzymatic roles. Therefore, these genes may serve as targets in future studies on CHSs in tobacco and their relationship to flavonoid biosynthesis and drought tolerance.

\section{Declarations}

\section{Conflicts of interest}

There are no conflicts to declare.

\section{Acknowledgements}

This work was supported by the Project of the Tobacco Genomic Program (No. 110201801031, JY-08; 110201601031, JY-05; 110201401012 JY-12).

\section{References}

1 E. Domínguez, L. España, G. López-Casado, J. Cuartero and A. Heredia, Funct. Plant Biol., 2009, 36, 613-620.

2 H. A. Stafford, Plant Physiol., 1991, 96, 680-685.

3 C. H. Shih, H. Chu, L. K. Tang, W. Sakamoto, M. Maekawa, I. K. Chu, M. Wang and C. Lo, Planta, 2008, 228, 1043-1054.

4 K. S. Gould, J. Biomed. Biotechnol., 2004, 2004, 314-320.

5 R. V. Phadke, A. Bhattacharyya, A. Handique, K. Jain, A. Kumar, V. Singh, D. Baruah, T. Kumar, S. Patwari and B. M. Mohan, Interv. Neuroradiol., 2014, 20, 357-367.

6 G. L. Wang, Z. Wei, S. X. He, X. J. Zhou and Z. S. Liang, Zhiwu Ziyuan Yu Huanjing Xuebao, 2011, 20, 1-8.

7 Y. Yuan, Y. Liu, C. Wu, S. Chen, Z. Wang, Z. Yang, S. Qin and L. Huang, PLoS One, 2012, 7, e42946.

8 J. L. Ferrer, J. M. Jez, M. E. Bowman, R. A. Dixon and J. P. Noel, Nat. Struct. Biol., 1999, 6, 775-784.

9 D. Y. Suh, K. Fukuma, J. Kagami, Y. Yamazaki, M. Shibuya, Y. Ebizuka and U. Sankawa, Biochem. J., 2000, 350, $229-235$.

10 J. M. Jez and J. P. Noel, J. Biol. Chem., 2000, 275, 39640-39646.

11 X. J. Liu, Y. N. Chuang, C. Y. Chiou, D. C. Chin, F. Q. Shen and K. W. Yeh, Planta, 2012, 236, 401-409.

12 B. Zhou, Y. Wang, Y. Zhan, Y. Li and S. Kawabata, Am. J. Bot., 2013, 100, 2458-2467.

13 L. Chen, H. Guo, Y. Lin and H. Cheng, Plant Cell Rep., 2015, 34, 885-894.

14 Y. Mo, C. Nagel and L. P. Taylor, Proc. Natl. Acad. Sci. U. S. A., 1992, 89, 7213-7217.

15 R. B. Horsch, J. E. Fry, N. L. Hoffmann, D. Eichholtz, S. G. Rogers and R. T. Fraley, Science, 1985, 227, 1229-1231.

16 K. Singh, J. Raizada, P. Bhardwaj, S. Ghawana, A. Rani, H. Singh, K. Kaul and S. Kumar, Anal. Biochem., 2004, 335, $330-333$.

17 X. Liu, S. Liu, J. Wu, B. Zhang, X. Li, Y. Yan and L. Li, Plant Physiol. Biochem., 2013, 70, 354-359.

18 M. Jiang and J. Zhang, Plant Cell Physiol., 2001, 42, 1265-1273.

Page 6/11 
19 K. V. Madhava Rao and T. V. S. Sresty, Plant Sci., 2000, 157, 113-128.

20 J. Y. Qian, D. Liu and A. G. Huang, Food Chem., 2004, 87, 283-288.

21 N. Sierro, J. N. D. Battey, S. Ouadi, N. Bakaher, L. Bovet, A. Willig, S. Goepfert, M. C. Peitsch and N. V. Ivanov, Nat. Commun., $2014,5,3833$.

22 S. Xu, T. Brockmöller, A. Navarro-Quezada, H. Kuhl, K. Gase, Z. Ling, W. Zhou, C. Kreitzer, M. Stanke, H. Tang, E. Lyons, P. Pandey, S. P. Pandey, B. Timmermann, E. Gaquerel and I. T. Baldwin, Proc. Natl. Acad. Sci. U. S. A., 2017, 114, 6133-6138.

23 The Tomato Genome Consortium, Nature, 2012, 485, 635-641.

24 H. T. M. Tran, T. Ramaraj, A. Furtado, L. S. Lee and R. J. Henry, Plant Biotechnol. J., 2018, 16, 1756-1766.

25 The Arabidopsis Genome Initiative, Nature, 2000, 408, 796-815.

26 A. Bateman, L. Coin, R. Durbin, R. D. Finn, V. Hollich, S. Griffiths-Jones, A. Khanna, M. Marshall, S. Moxon, E. L. L. Sonnhammer, D. J. Studholme, C. Yeats and S. R. Eddy, Nucleic Acids Res., 2004, 32, D138-D141.

27 C. B. Do, M. S. P. Mahabhashyam, M. Brudno and S. Batzoglou, Genome Res., 2005, 15, 330-340.

28 S. Guindon, F. Delsuc, J. F. Dufayard and O. Gascuel, in Bioinformatics for DNA Sequence Analysis, ed. D. Posada, Humana Press, Totowa, NJ, 2009, pp. 113-137.

29 I. Abe and H. Morita, Nat. Prod. Rep., 2010, 27, 809-838.

30 S. S. Kim, E. Grienenberger, B. Lallemand, C. C. Colpitts, S. Y. Kim, C. d. A. Souza, P. Geoffroy, D. Heintz, D. Krahn, M. Kaiser, E. Kombrink, T. Heitz, D.-Y. Suh, M. Legrand and C. J. Douglas, Plant Cell, 2010, 22, 4045-4066.

31 K. Abdel-Lateif, V. Vaissayre, H. Gherbi, C. Verries, E. Meudec, F. Perrine-Walker, V. Cheynier, S. Svistoonoff, C. Franche, D. Bogusz and V. Hocher, New Phytol., 2013, 199, 1012-1021.

32 A. P. Wasson, F. I. Pellerone and U. Mathesius, Plant Cell, 2006, 18, 1617-1629.

33 Z. Chen, J. Tan, G. Yang, M. Miao, Y. Chen and T. Li, Phytochem. Lett., 2012, 5, 233-235.

34 J. Chen, H. Leng, Y. Duan, W. Zhao, G. Yang, Y. Guo, Y. Chen and Q. Hu, Phytochem. Lett., 2013, 6, 144-147.

35 R. Mittler, S. Vanderauwera, M. Gollery and F. Van Breusegem, Trends Plant Sci., 2004, 9, 490-498.

36 C. Rice-Evans, N. Miller and G. Paganga, Trends Plant Sci., 1997, 2, 152-159.

37 M. Melidou, K. Riganakos and D. Galaris, Free Radic. Biol. Med., 2005, 39, 1591-1600.

38 G. Agati and M. Tattini, New Phytol., 2010, 186, 786-793.

39 L. Jaakola and A. Hohtola, Plant Cell Environ., 2010, 33, 1239-1247.

40 R. S. Dhindsa and W. Matowe, J. Exp. Bot., 1981, 32, 79-91.

\section{Figures}


B nptII attR1 NtCHS attR2 CaMV3 S.P EGFP CaMV3 S.P $\quad$ B
$\mathrm{C} 1 \quad \mathrm{C} 2 \quad \mathrm{C} 3$
T1 $\quad$ T2 $\quad$ T3

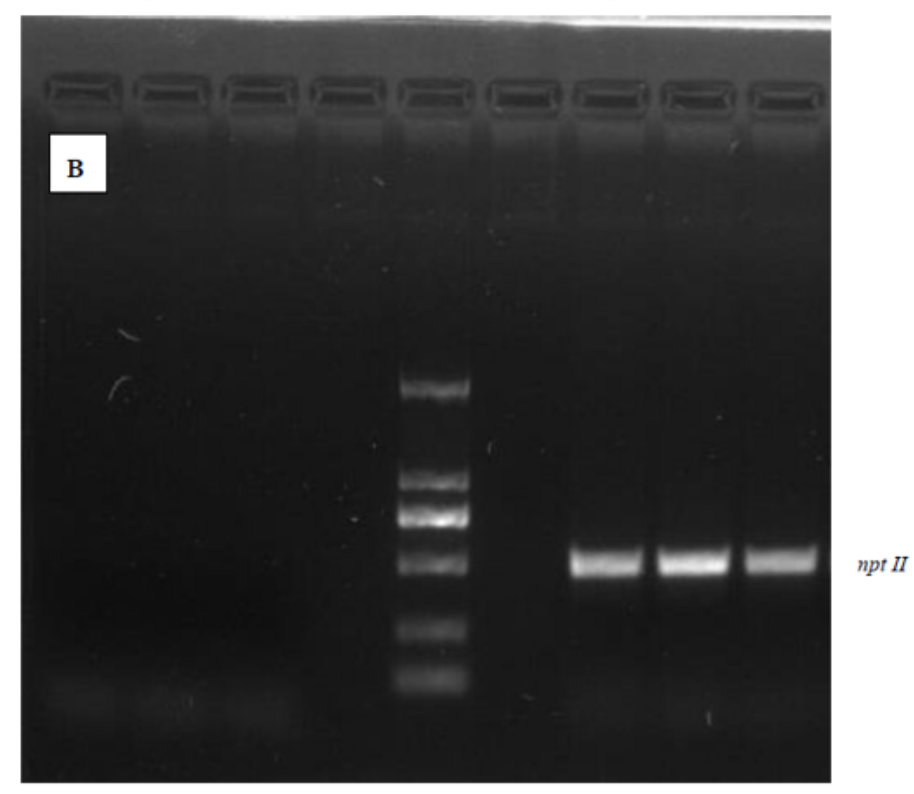

\section{Figure 1}

Schematic map of the tDNA region of pH7WG2D-NtCHS and confirmation of successful transformation. (a) schematic representation of ph7wg2d harboring the ntchs cdna. (b) pcr using genomic DNA as the template confirms the introduction of npt II into the transgenic tobacco line. Npt II is absent in the control tobacco lines, C1, C2, and C3. 

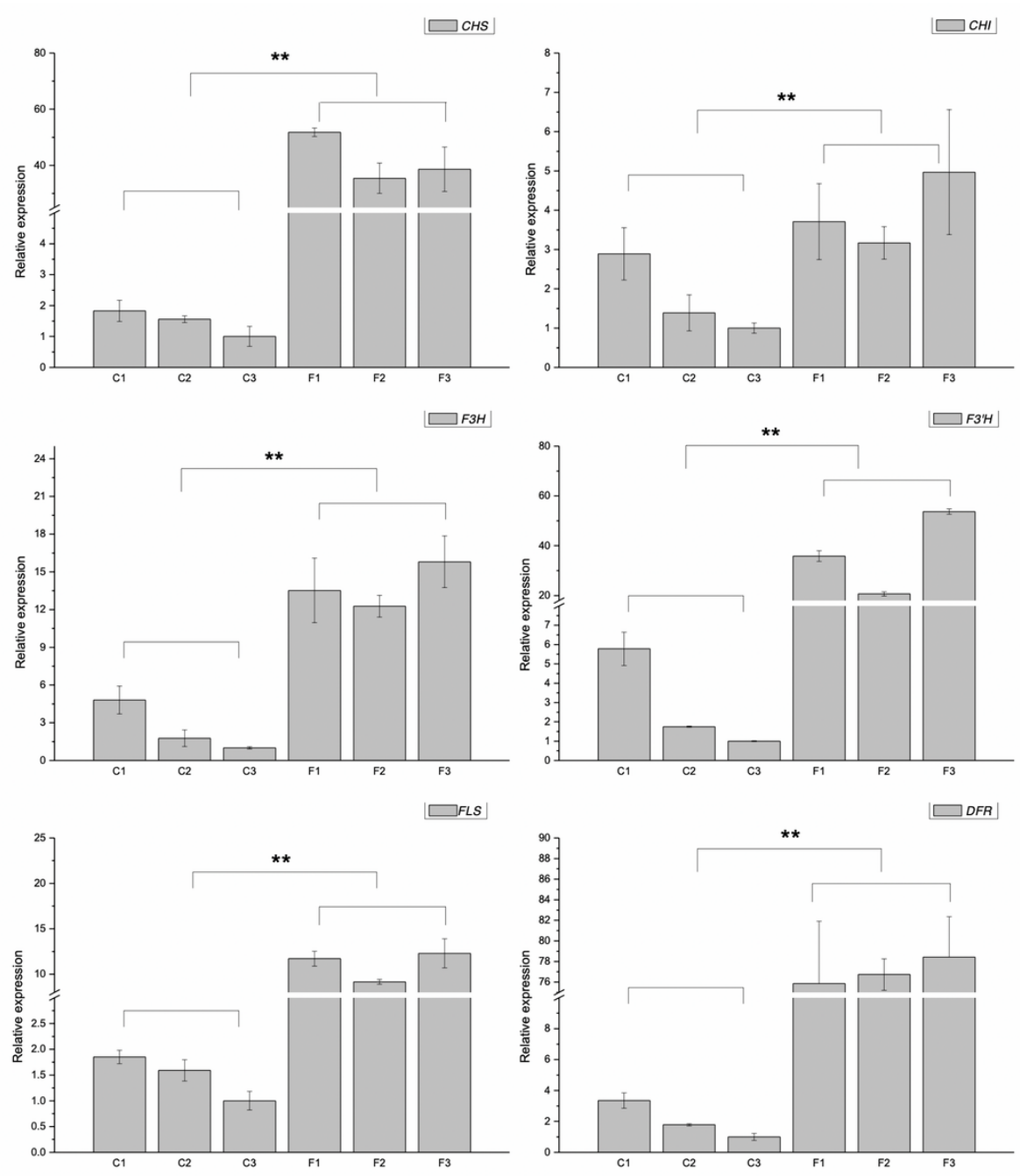

\section{Figure 2}

Abundance of the transcripts of flavonoid transcriptional genes in transgenic and control tobacco lines measured by RT-qPCR: (a) CHS, (b) CHI, (c) F3H, (d) $\mathrm{F}^{\prime} \mathrm{H}$, (e) FLS, and (f) DFR. * $\mathrm{p}<0.05 ;{ }^{* *} \mathrm{p}<0.01$.

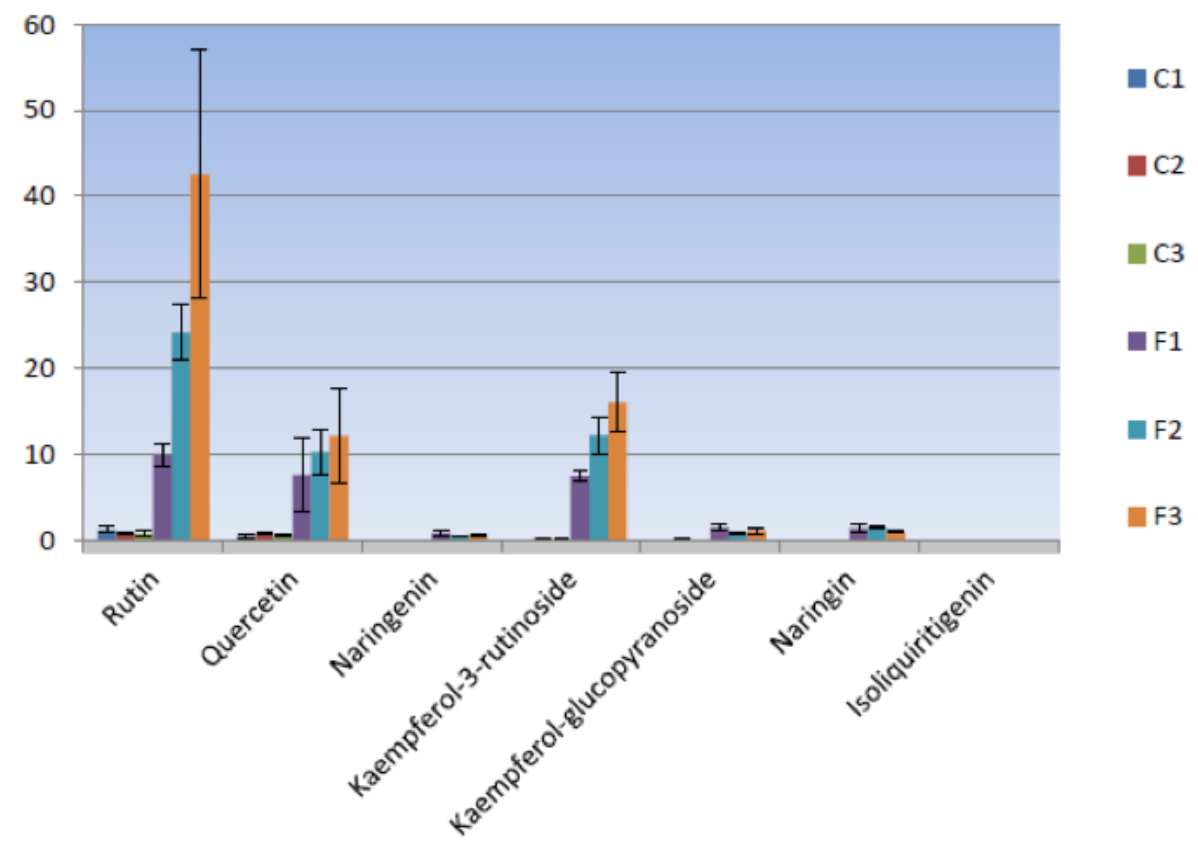


Quantification of seven flavonoids in transgenic and control tobacco lines by HPLC-MS ESI+.

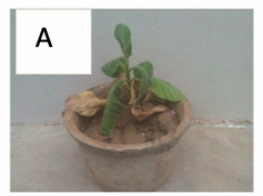

C1

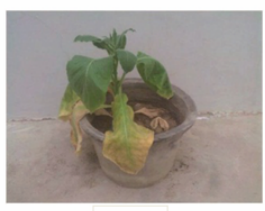

F1

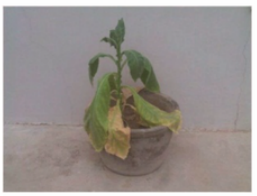

C2

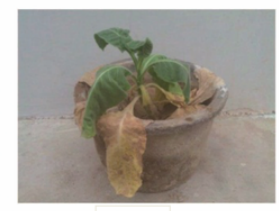

F2

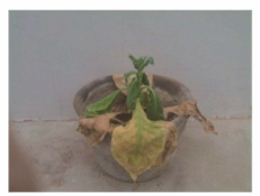

C3

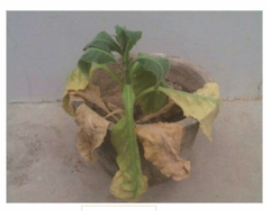

F3
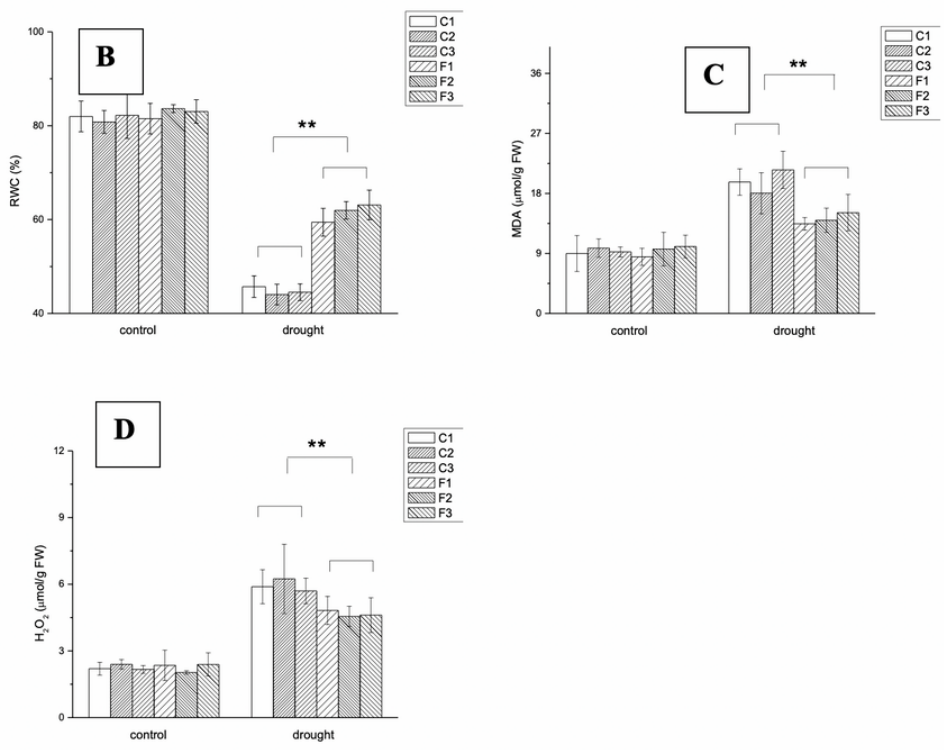

\section{Figure 4}

(a) Qualitative observations of the drought experiment. (b) RWC in transgenic and control tobacco lines. (c) Concentrations of MDA in transgenic and control tobacco lines. (d) Concentrations of $\mathrm{H} 2 \mathrm{O} 2$ in transgenic and control tobacco lines. C1-C3: control tobacco lines; F1-F3: T1 generation transgenic tobacco lines. ${ }^{*} p<0.05$; ${ }^{\star *} p<0.01$. 
A

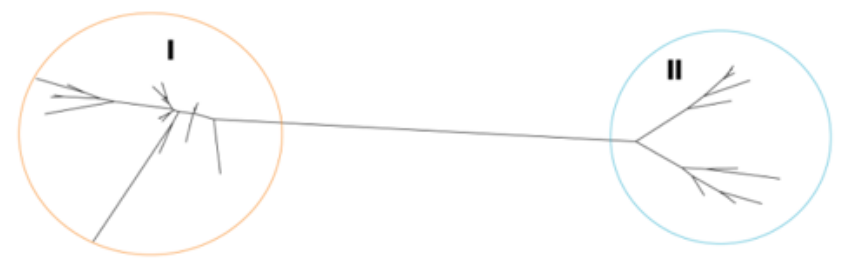

B

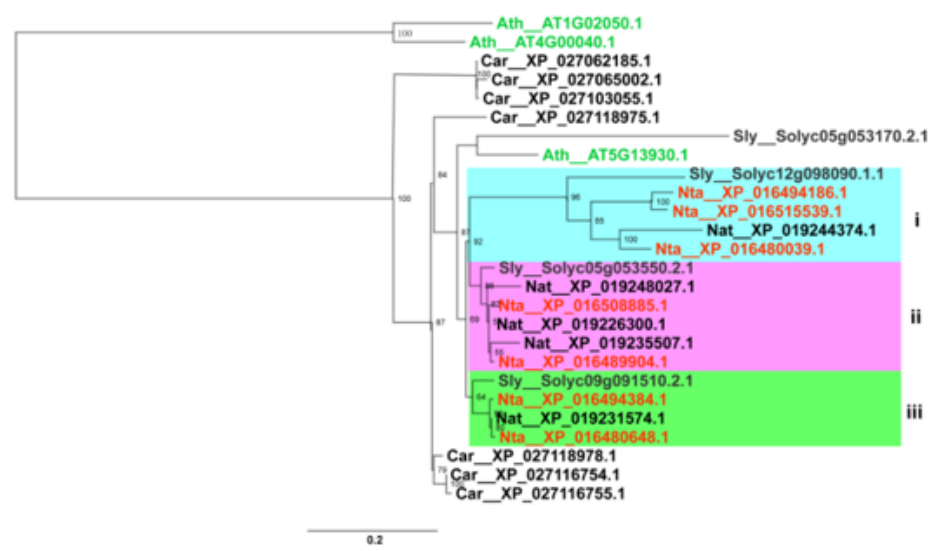

\section{Figure 5}

Phylogeny of CHS family peptides. CHS family peptide sequences were retrieved from tobacco, tomato, Arabidopsis, coffee, and N. attenuata. PhyML was used for the reconstruction of phylogenetic trees. (a) Unrooted phylogenetic tree of CHS family genes showing two independent groups (circled and labeled with I and II, respectively). (b) Phylogenetic tree of CHS genes. Red labels indicate tobacco genes, green labels indicate Arabidopsis genes. Clades $\mathrm{i}$, ii, and iii are shaded in light green, purple, and green, respectively.

A

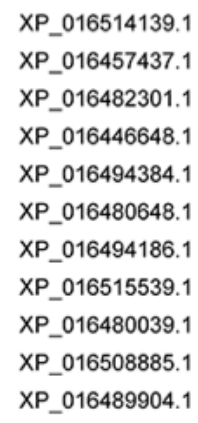

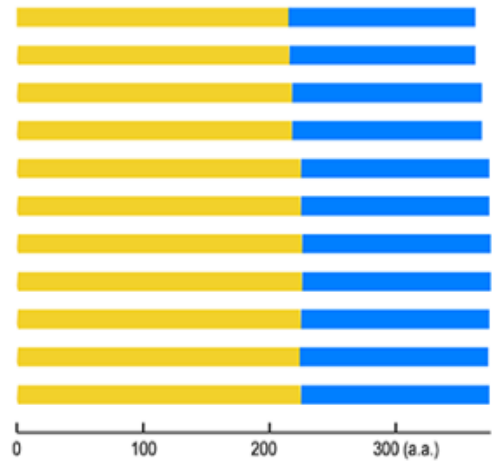

B

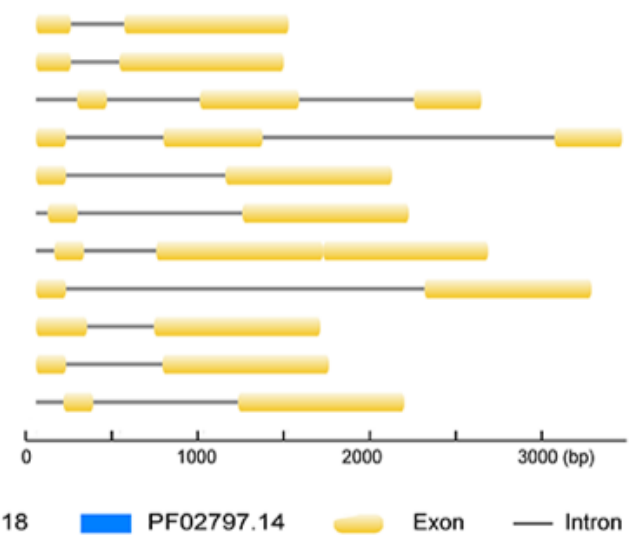

Figure 6

Domain and gene structures of tobacco CHS genes. (a) Domain structures of the tobacco CHS peptides showing two conserved domains (yellow and blue). (b) Exon-intron structures of the tobacco CHS genes showing exons (yellow boxes) and introns (lines).

\section{Supplementary Files}

This is a list of supplementary files associated with this preprint. Click to download.

- Supplementary.docx 